\title{
Armando Petrucci
}

\section{"L'antiche e le moderne carte": imitatio e renovatio nella riforma grafica umanistica*}

1. Fra il 1277 e il 1280 Cimabue raffigura nella crociera della chiesa superiore di Assisi, per commissione di papa Nicolò III Orsini, gli evangelisti e le rispettive regioni evangelizzate (l'Italia, la Grecia e così via), identificate per mezzo della rappresentazione della città capitale di ciascuna di esse e per mezzo del nome, dipinto in lettere maiuscole gotiche grandi, massicce, ricche di elementi ornamentali ${ }^{1}$.

L'Italia è simboleggiata da un fantastico agglomerato di monumenti romani; fra gli altri, spicca il Pantheon, sul cui frontone si scorge un'iscrizione dipinta in lettere appena tracciate. Si è affermato che tali lettere constituirebbero la prima restituzione della capitale classica epigrafica nella pittura del Medioevo europeo ${ }^{2}$; ma a mio avviso questa interpretazione va rovesciata nel suo esatto contrario: queste lettere (QULT), quasi invisibili, testimoniano in realtà soltanto l'impossibilità del ritorno al passato e di una vera e consapevole restaurazione grafica; e si risolvono nella denuncia di una traumatica rottura con la tradizione antica. Poco tempo prima, del resto, il grande giurista bolognese Odofredo aveva confessato le difficoltà che si incontravano nella lettura delle iscrizioni romane classiche $e$ in particolare nella comprensione delle abbreviazioni ${ }^{3}$.

In effetti, lo si sa bene, il XIII secolo costituisce un periodo di totale rinnovamento nel dominio della scrittura latina e del libro manoscritto europeo; periodo che corrisponde a un moto generale di vera e propria (e consapevole) negazione della tradizione grafico-libraria antica e delle sue sopravvivenze altomedievali. Ormai in tutta

- La volontà di mantenere a questo contributo la natura di semplice comunicazione ad un seminario e la impossibilità di rielaborarlo in forma più ampia per mancanza di tempo e di spazio, mi hanno indotto a lasciare il testo più o meno nella forma in cui è stato recitato; e perciò anche a rinunciare ad ogni apparato iconografico, del resto facilmente surrogabile per chiunque, sulia base della bibliografia citata.

' Si veda per questo M. Andaloro, Ancora una volia sull'Ytalia di Cimabue, in Arte medievale, 2 (1984), pp. 143-181 (con belle riproduzioni: cf. in particolare pp. 145, 161-162).

${ }^{2}$ Così E. Battisti, Cimabue, Milano 1963, p. 86, n. 62: .Dalla riproduzione fotografica si constata che Cimabue, per la prima volta in pittura, ha anche ripreso la capitale classica, riproducendo con assoluta fedeltà l'epigrafe dedicatoria del Pantheon, ma, a quanto intravvedo, cambiandola in un elogio di Augusto.

${ }^{3}$ Cf. I. Calabi Limentani, Sul non saper leggere le epigrafi classicbe nei secoli XII e XIII; sulla scoperta graduale delle abbreviazioni epigrafiche, in ACME, XXIII (1970), pp. 253-282, in particolare pp. 255-257. 
l'Europa i libri vengono scritti in una minuscola del tutto nuova come tecnica di esecuzione, in quanto il tessuto grafico risulta scomposto in una serie coordinata di tratti di penna giustapposti; si leggono libri nuovi, caratterizzati da una impaginazione complessa, a colonne e a maglie serrate; e questi libri vengono prodotti, commerciati e conservati secondo prassi assai diverse rispetto a quelle del passato. Anche le iscrizioni presentano un aspetto nuovo, sia nella scrittura, una gotica maiuscola fitta e serrata, sia nell'impaginazione, sia, infine nel colore: poiché spesso esse hanno la superficie dorata.

Si può dunque a mio parere legittimamente affermare che il Duecento rappresentò una vera e propria rivoluzione grafica che investi, sia pure in tempi e in modi leggermente diversi, tutti i paesi dell'Europa occidentale.

2. Il processo di rinnovamento, o meglio di modificazione totale della forma-libro, che caratterizzò il mondo della cultura gotica, investì naturalmente anche i manoscritti contenenti testi di autori classici. In particolare, a partire dall'inizio stesso del secolo XII, nella tradizione iconografica relativa ai temi e ai personaggi del mondo classico si assistette ad una trasposizione totale delle figurazioni e delle rappresentazioni dalle forme tradizionali a forme modeme, o meglio contemporanee ai singoli pittori; il che comportò un processo globale di modernizzazione dell'iconografia classica e delle sue sopravvivenze carolingie, di cui si era perduta o si era, meglio, rifiutata la memoria storica.

Nel 1933 Erwin Panofsky e Fritz Saxl hanno identificato e posto nel giusto rilievo questo processo di edecomposition of classical representation.4; dopo la Rinascenza carolingia, essi affermano, eclassical subject matter and classical form were separated.5, perché •medieval western art was unable or ... was unwilling to retain a classical prototype without destroying either its original form or its original meaning. ${ }^{6}$. In particolare, per quanto riguarda l'iconografia degli dei del pantheon antico, Jean Seznec ha potuto rilevare, a partire appunto dall'inizio del XII secolo, un analogo e sorprendente processo di stravolgimento e di modernizzazione ${ }^{7}$.

Ma il processo di trasformazione del modello dei libri con testi classici non si limitò all'iconografia ed investì l'aspetto generale dei codici: già nel XII, ma ancor più nel secolo seguente, i testi di Virgilio, di Lucano, di Ovidio, di Livio, di Boezio, di Sallustio e così via assunsero il formato, la struttura, l'impaginazione, il sistema di partizione interna, i colori e l'ornamentazione dei coevi libri della cultura universitaria e scolastica, dei libri scritturali e patristici, delle opere di san Tommaso, di quelle di medicina o di diritto.

4 E. Panofsky - F. Saxl, Classical Mythology in medieval Art, in Metropolitan Museum Studies, 4 (1932-33), pp. 228-280 (la citazione da p. 238).

s Ibid., p. 237.

'Ibid., p. 228.

'J. Seznec, The Survival of the pagan Gads. The Mytbological Tradition and its Place in Rinaissance Humanism and Art, Princeton 1972, in particolare pp. 156-167. 
3. In particolare in Italia si assistette nel corso del Trecento alla nascita di un tipo di libro manoscritto miniato contenente testi di autori classici che si può bene a ragione definire un vero e proprio libro -cortese. Si tratta infatti di grandi manoscritti membranacei di lusso, caratterizzati dalla scrittura gotica rotonda e massiccia disposta su due colonne, dalla disposizione spessa del testo sulla linea e nello spazio complessivo della pagina, dalle rubriche e dai titoli in rosso, dall'ornamentazione a viticci o a fogliami, dalle miniature a vignetta, disposte nei margini o all'interno del testo.

A proposito del processo di modemizzazione dell'iconografia classica che caratterizza questo tipo di libro manoscritto, assai recentemente Jeanne Courcelle ha osservato che exception faite des manuscrits les plus tardifs, tous les personnages évoluent dans les décors de l'époque: paysages, architectures, intérieurs, évoquent les pays et les mours du peintre. On aboutit ainsi à une christianisation inconsciente de certaines épisodes .... ${ }^{8}$; ed ha anche parlato di illustrateurs dénoués de toute culture antique. 9 . $\mathrm{Ma}$ in realtà non tanto o non solo di ignoranza si trattava, quanto piuttosto della necessità di raccordare al presente, attraverso il processo interpretativo ed esplicativo rappresentato dall iconografia, testi nati in un passato troppo lontano e difficilmente comprensibile per $i$ lettori o per i possessori (non sempre coincidenti) dei libri che li contenevano.

Il fenomeno ebbe un'estensione europea; tutti conosciamo le fiorite trasposizioni anacronistiche del gotico internazionale, gli imperatori con lussuosi vestiti del Trecento, le matrone coperte di lunghi abiti colorati, i guerrieri con armature medievali, gli dei trasformati in filosofi, e così via. In Italia, per quanto riguarda il libro manoscritto, il fenomeno si diffuse largamente soprattutto nelle aree proprie della cultura cortese, e cioè nelle grandi corti padane, nel Veneto e nella Napoli angioina ${ }^{10}$; $\mathrm{e}$ inoltre trovò un supporto letterario e un terreno di proliferazione adatti nei volgarizzamenti di testi classici o nelle rielaborazioni volgari di storie classiche che riempivano le biblioteche dell'aristocrazia contemporanea e circolavano ampiamente anche in ambienti borghesi e "popolari," Ma è difficile precisare, in assenza di studi particolari e di censimenti specifici di codici miniati, quali aree testuali e quali autori furono maggiormente investiti dal fenomeno; certamente gli storici (Livio, Valerio Massimo, Sallustio, per esempio e fra i primi) e le compilazioni storiche; ma poi anche poeti come Virgilio e Lucano; e ancora Plinio, Terenzio, e così via.

4. E' legittimo a questo punto porsi la domanda di come nel panorama finora descritto si collocassero la figura di Francesco Petrarca e l'opera di recupero dei testi classici da lui avviata. Petrarca conosceva bene la differenza fra le antiche e le mo-

${ }^{B} \mathrm{~J}$. Courcelle, Les illustrations de l'Enéide dans les manuscrits $d u X^{\mathbf{e}}$ au $X V^{\mathbf{e}}$ siècle, in Lectures médiériales de Vingile..., Rome 1985, p. 400.

9 Ibid., p. 401.

${ }^{10} \mathrm{Per}$ Napoli in particolare, si veda A. Perriccioli Saggese, I romanzi cavallereschi miniali a Na. poli, Napoli 1979, con un gran numero di illustrazioni.

"Cf. per questo A. Petrucci, Le biblioteche antiche, in Letteratura italiana, a cura di A. Asor Rosa, II, Praduzione e consumo, Torino 1983, pp. 540-546. 
derne carte ${ }^{12}$, cioè fra i testi e $\mathrm{i}$ libri del passato e quelli del mondo a lui contemporaneo. Secondo il giudizio di Guido Martellotti, per Petrarca il desiderio di avvicinare il modemo all'antico implica insieme la coscienza di una distanza da colmare; e la coscienza di tale distanza è principio e fonte di un intendimento storico. ${ }^{13}$, d'una vera $e$ propria coscienza della storia.

Petrarca, lo si sa bene, ha condotto una polemica accanita contro la scrittura testuale gotica del suo tempo, che egli giudicava eccessivamente artificiale, e contro i fondamenti stessi del sistema produttivo del libro contemporaneo ${ }^{14}$. Ma a proposito del suo Livio, Albinia de la Mare ha giustamente osservato che in the early fourteenth century Petrarch and Landolfo Colonna had compressed all three decades into one volume, in the way that the scribes of the thirteenth century had learned to treat the major works of the Fathers. ${ }^{13}$. Ed è proprio il codice Par. lat. 5690 (che peraltro, oltre Livio, tramanda anche Ditti Cretese e Floro), già eseguito per Landolfo Colonna e quindi posseduto e annotato dal Petrarca, che contiene uno splendido e ricchissimo ciclo illustrativo completamente modernizzato ed articolato in trenta vignette molto abilmente inserite nei margini e all'interno delle lettere iniziali ${ }^{16}$. D'altra parte, anche il suo caro

${ }^{12}$ Così nella canzone 28 a Giacomo Colonna, v. 77.

${ }^{13}$ Cosi nella prefazione a Francesco Petrarca, Prase, Milano-Napoli 1955, p. XII.

${ }^{14} \mathrm{Cf}$. per questo A. Petrucci, Libro e scrittura in Francesco Petrarca, in Libri, scrittura e pubblico nel Rinascimento. Guida storica e critica, a cura di A. Petrucci, Bari 1979, pp. 3-20, con rinvio alla letteratura precedente, e in particolare ad A. Petrucci, La scrittura di Francesco Petraria. Citta del Vaticano 1967 (Studi e Testi, 248).

is A. de la Mare, Florentine Manuscripts of Liiy in the fifteenth Century; in Lity; a cura di T. A. Dorey, London 1971, p. 186.

16 Intorno a questo codice, alla sua datazione, alla sua formazione e alla sua origine molte e discordi sono state negli ultimi decenni le proposte e le ipotesi, che quasi sempre si sono ignorate a vicenda (per la bibliografia anteriore al 1967 si veda Petrucci, La scriftura di Francesco, cit., p. 125, n. 37); dalla attribuzione sicura e unitaria ad Avignone, sostenuta da G. Billanovich (e ribadita in G. Billanovich, La tradizione del testo di Livio e le origini dell'Umanesimo. Tradizione e fortuna di Livio tra Medioevo e Umanesimo, p. I, Padova 1981, pp. 132, 189-191; e in Id., La biblioteca papale salvò le Storie di Litio, in Studi petrarcheschi, III (1986), p. 43, all'attriburione a Salemo di B. Degenhart e A. Schmitt (Corpus der italieniscben Zeichnungen. 1300-1450. Süd- und Miltelitalien, II, 1, p. 108; II, 2, figg. 402, 406, ecc.), a quella dell'articolazione del manoscritto in due parti distinte, l'una attribuibile alla fine del Duecento e napoletana, l'altra avignonese e dei primi del Trecento, affacciata da F. Bologna (cf. di lui Il . Tito Livio. n. 5690 della B. ${ }^{\prime}$ di Parigi. Miniature ericerche proto-umanistiche tra Napoli e Alignone alle saglie del Trecento: constatazioni e ipoteri ion appendice iconografica, in Gli Angioini di Napoli e di Ungheria, Roma, Accademia Nazionale dei Lincei, 1974 (Quademi attuali di scienza e cultura, 210), pp. 41-116), per arrivare all'ultima in ordine di tempo, di F. Avril, che riconosce la composizione in più tempi del codice, ma lo attribuisce ad un unico centro, Roma, e ad un unico momento: primissimi del Trecento e comunque non dopo il 1309 (Dix siècles d'enluminure italienne (VI ${ }^{e}-X V I^{e}$ siècle), Paris, Bibliothèque Nationale, 1984 , p. 50, n. 39). E si vedano ancora due contributi particolari, ma utili: F. Sabatini, Napoli Angioina. Cultura e società, Napoli 1974, Pp. 37-38; e F. Bruni, Un documento sul Livio napole. tano-aragonese del Petrarca, oggi Par. lat. 5690, in Medioevo romanzo, IV (1977), pp. 341-349 (con l'ardita e difficilmente sostenibile identificazione del codice stesso con un Livio ricordato in una lettera di Giacomo Il d'Aragona come in vendita a Napoli nel 1314 per 100 fiorini). Il manoscritto è effettivamente diviso in due parti (I: cc. 1-168; II : cc. 169-368), diverse per testi contenuti, per mani di scribi (due per la prima parte, con passaggio fra c. $41 v$ e c. $42 \mathrm{r}$; più d'una per la 
Virgilio Ambrosiano, con le illustrazioni emodernizzanti, di Simone Martini, è l'esempio perfetto d'un libro eseguito secondo i modelli moderni; e ancora, le iscrizioni funerarie dal Petrarca composte per il nipote Francescuolo nel 1368 (oggi a Pavia) sono state incise in ricche maiuscole di puro stile gotico ${ }^{17}$.

In effetti il rapporto del Petrarca con l'antichità classica e i suoi modelli era regolato da una teoria dell'imitazione insieme flessibile e libera, che egli enunciò in una letteraprogramma inviata da Pavia a Boccaccio nel 1366, precisando il suo personale concetto di una similitudo latens: : Curandum imitatori ut quod scribit simile, non idem sit ....; aln quibus, cum magna sepe diversitas sit membrorum, umbra quedam et quem pictores nostri aerem vocant ... similitudinem illam facit ....; e ancora: :illa enim similitudo latet, hec eminet; illa poetas facit, hec simias. ${ }^{18}$. In conclusione, occorre ammettere che nella sua graduale elaborazione di una nuova norma grafico-libraria, Petrarca non sorpassò mai i limitj di questa sua cumbras, per andare al di là di una personale ed equilibrata operazione di semplice esimilitudo. ${ }^{19}$.

5. E' probabilmente vero che nella storia della cultura scritta non esistono cambiamenti o innovazioni, anche radicali, che non si ispirino a modelli anteriori eventualmente di secoli. Ciò si verificò anche a proposito del tipo di libro, del tutto nuovo, che Niccolò Niccoli e Poggio Bracciolini, aiutati da Coluccio Salutati, elaborarono e produssero a Firenze fra gli ultimi anni del XIV secolo e i primi anni del secolo seguente; un libro, come ben si sa, che voleva essere, e che in buona parte era, una riproduzione puntuale fino nei particolari e nei sistemi di fattura dei manoscritti prodotti in Italia dall'XI all'inizio del XII secolo; di essi, infatti, si riproducevano, attraverso un vero e proprio procedimento di imitatio puntuale, il formato, l'impaginazione, i sistemi e i tipi di rigatura, l'omamentazione e infine, o meglio prima di tutto, la scrittura: quella santiqua. .castigata et clara, ${ }^{20}$, che fu riprodotta nelle sue forme in modo quasi fotografico $^{21}$.

seconda; tutte italiane) e per tipo di rigatura, doppia nella prima parte, semplice nella seconda. Ma le mani sembrano coeve; $e$ inoltre esistono due elementi coevi di continuità: il sistema dei richiami di fine fascicolo, posti regolarmente a destra, sotto la seconda colonna dell'ultima facciata, e la numerazione delle vignette, eseguita certamente da mano contemporanea in cifre arabiche, che va da 12 (c. 179r) a 29 (c. 358v) e che presuppone un'unitarietà della serie, probabilmente eseguita in un medesimo periodo e numerata a fini di pagamento degli esecutori. In conclusione, a mio avviso il codice sarebbe stato eseguito agli inizi del secolo XIV in tempi leggermente diversi da più copisti e artisti italiani per un committente unico in un medesimo luogo; ma per quanto riguarda l'origine, oggi non so né formulare un'ipotesi, né accettare incodizionatamente quelle finora affacciate da altri.

17 Per questo e per il rapporto con Simone Martini, cf. Petrucci, La scrittura di Francesco, cit., pp. 58-70.

${ }^{18}$ F. Petrarca, Le Familiari, IV, a cura di U. Bosco, Firenze 1942, p. 206; sul passo, cf. le acute osservazioni di E. H. Gombrich, The Style all'antica: Imitation and Assimilation, in Id., Norm and Form. Studies in the Art of the Renaissance, London-New York 1971, pp. 122-128.

${ }^{19} \mathrm{Cf}$. ancora Petrucci, La scrittura di Francesco, cit., pp. 61, 68-70.

${ }^{20}$ Famosa definizione del Petrarca, per cui Petrucci, La scrittura di Francesco, cit., p. 66.

${ }^{21} \mathrm{Cf}$. A. Petrucci, Anticamente maderni e modernamente anticbi, in Libri, scrittura e pubblico, cit., pp. 21-36. 
Già Emst H. Gombrich, in un articolo ben noto del 1967, ha posto in rilievo che esiste un parallelismo evidente fra l'imitatio grafica dei calligrafi dilettanti amici del Niccoli e l'architettura di Filippo Brunelleschi, poiché l'una e l'altra forma d'imitazione sono fondate su uno stretto rapporto con modelli di età romanica: .... quali che fossero le intenzioni di Brunelleschi, la ricerca moderna ha rivelato che, al pari degli umanisti, egli derivò la propria alternativa al gotico più da esemplari pregotici presenti a $\mathrm{Fi}$ renze ... che da uno studio delle rovine romane ....; e ancora: . Al pari della riforma della scrittura, la riforma dell'architettura fu certo dovuta al nuovo ed esclusivo entusiasmo per l'antichità, ma la sua ispirazione derivò principalmente dai monumenti del passato fiorentino che erano venerati come reliquie romane. ${ }^{22}$.

A questo punto occorre sottolineare con forza un fatto di grande importanza per il nostro assunto: nei manoscritti del Niccoli o in quelli di Poggio Bracciolini si trova l'santiquas, si trova una nuova (o meglio antica) formula di impaginazione (al di sopra della prima linea di rigatura), si trovano le iniziali a bianchi girari; ma non si troveranno mai miniature o comunque illustrazioni. Albinia de la Mare lo ha già posto in rilievo a proposito di Livio: .Despite the opportunities which his text offered for illustration, it seems that Livy was generally considered in Italy as a serious author, not to be treated as a picture book ....23; ma il fenomeno è generale e investe non soltanto le scelte dei programmi iconografici, bensi anche quelle dei modelli e dei procedimenti grafici.

6. Le regole e $\mathrm{i}$ procedimenti della pratica dell'imitatio grafica umanistica sono enunciati da Ambrogio Traversari in una lettera inviata nel 1432 a suo fratello Gerolamo; in essa egli lo esorta a epriscam illam in scribendo imitari puritatem ac suavitatem. Quod tunc - aggiunge - adsequare facilius si ex emendatissimo antiquoque codice quidpiam tibi transcribendum deligas totoque annisu ad unguem exemplar fidum imitari, (forse per imiteris, secondo la Rizzo) ${ }^{24}$.

In questa lettera, definita da Silvia Rizzo *quasi il manifesto della riforma umanistica della scrittura ${ }^{25}$, si ha, probabilmente per la prima volta, la chiara formulazione di un programma di imitazione grafica, o meglio di una pratica imitativa; che peraltro si limita a riprodurre il disegno delle lettere, e non il loro tratteggio, e che utilizza gesti, prassi, strumenti e tecniche esecutive che sono, o possono essere, assai diversi da quelli propri dei modelli di età carolingia o carolina.

In effetti, nell'antiqua, italiana della prima metà del secolo XV il tratteggio e il disegno delle lettere e le tecniche di esecuzione della scrittura differivano grandemente a causa della diversità dei modelli e delle profonde differenze nell'educazione grafica degli scribi. Già nel corso dei primi dieci anni del XV secolo (Albinia de la

${ }^{22}$ E. H. Gombrich, Dalla rinascita delle lettere alla riforma delle arti: Niccolo . Vicoli e Filippo Brunelleschi, in Id., L'eredità di Apelle. Studi sull'arte del Rinascimento, Torino 1986, pp. 140 e 141

${ }^{23}$ A. de la Mare, Florentine Manuscripts, cit., pp. 187-188.

${ }^{24}$ A. Traversari, Latinae epistolace ..., a cura di P. Canneti - L. Mehus, II, Florentiae 1759, Ep. 385, col. S01; e S. Rizzo, $1 l$ lessico filologico degli umanisti, Roma 1973 (Sussidi eruditi, 26), p. 143.

2s Rizzo, Il lessico, cit., p. 143 
Mare l'ha dimostrato in un articolo celebre $)^{26}$ sono individuabili numerose interpretazioni di slittera antiqua, caratterizzate da diverse tecniche esecutive, da diverse temperature della penna, dall'imitazione di diversi modelli, con risultati grafici ed estetici assai differenti fra loro; si pensi, ad esempio, alla tipizzazione delle corsiveggianti -literulae, segnalate da Emanuele Casamassima nel $1974^{27}$, o alla scrittura grecizzante di Ciriaco Pizzicolli d'Ancona e dei suoi imitatori ${ }^{28}$. Il fenomeno investi anche le maiuscole, all'interno delle quali è possibile riconoscere diverse varietà stilistiche, influenzate ora, come quelle fiorentine e toscane, dai modelli dei manoscritti e delle epigrafi romanici dell'XI e del XII secolo ${ }^{29}$, ora, come in Lombardia e anche altrove, da modelli epigrafici e manoscritti bizantini o bizantineggianti; ma quest'ultimo è argomento degno di approfondimento particolare.

Questo processo di imitazione formale e differenziato di modelli grafici anteriori di qualche secolo condusse entro la prima metà del Quattrocento alla formulazione di una estetica della nuova scrittura basata essenzialmente su ideali assai generali, e generici, di equilibrio e di armonia. In particolare, l'umanista senese Mariano Sozzini, in un testo recentemente commentato da Fabio Troncarellij ${ }^{30}$, esprimeva nel 1440 tali ideali con chiarezza assoluta:

1) :Literarum corpora seu caracteres debita ac legitima forma componendi sunts: il che attiene al disegno delle singole lettere, che va tracciato seguendo un modello alegittimo:;

2) .Partes ad invicem distantes condecenti ac equali spatio disiungendae *: il che si riferisce alla necessaria spaziatura fra lettera e lettera e fra parola e parola che caratterizza la eantiqua. rispetto alla gotica;

3) *Aequalitatem ... ex superiori atque inferiori parte servare convenit, ne una alteram longitudine excedat, aut alia propter nimiam brevitatem ab altera excedatur .... con il che si prescrive l'uguaglianza delle aste ascendenti e discendenti componenti le singole lettere, al fine di evitare qualsiasi disarmonia;

4) per concludere con l'esaltazione di un principio generale di armonia e di equilibrio grafici: eea quae literarum aspectui plurimum amica, grata atque iocunda est: aequalitas. ${ }^{31}$

${ }^{26}$ A. de la Mare, Humanistic Script: the first ten Years, in Das Verbältnis der Humanisten zum Buch, a cura di F. Kraft - D. Wuttke, 1977, pp. 89-110.

${ }^{27}$ E. Casamassima, Literulae Latinae. Nota paleografica, in S. Caroti - S. Zamponi, Lo scrittoio di Bartolomeo Fonzio umanista fiorentino, Milano 1974, pp. IX-XXXIII.

${ }^{28} \mathrm{D}$. Fava, La scrittura libraria di Ciriaco d'Ancona, in Scritti di paleografia e diplomatica in onore di $V$. Federici, Firenze 1945, pp. 295-305.

${ }^{29} \mathrm{Cf}$. E. Casamissima, Lettere antiche, Note per la storia della riforma grafica umanistica, in Gutenberg Jahrbuch (1964) p. 25, n. 37.

${ }^{30} \mathrm{~F}$. Troncarelli, Grata et iocunda est aequalitas: Mariano Sozzini Ira Medioevo e Umanesimo, in La città dei segreti: magia, astrologia e cultura esoterica a Roma (XV-XVIII), Milano 1985, pp. S5-69.

${ }^{31}$ Troncarelli, Grata et iocunda. cit., p. 55. 
Tuttavia l'influenza diretta e reale di questi principi ideali sulla pratica quotidiana della scrittura e della fattura dei manoscritti umanistici doveva essere, a mio parere, assai modesta. Oltre alle diversità, già ricordate, riguardanti le stilizzazioni grafiche, ve ne erano infatti altre che investivano $i$ processi di fabbricazione e di preparazione dei codici; infatti l'istaurazione di una scrittura nuova non comportò automaticamente $e$ dappertutto il rinnovamento anche delle prassi produttive. Albert Derolez ha recentemente dimostrato che gli elementi di imitazione delle prassi di fattura carolingie introdotte nei codici umanistici dalla ostinata mania del Niccoli, furono rapidamente abbandonati dagli scribi professionisti; mi riferisco soprattutto alla disposizione della scrittura al disopra della prima riga, alla posizione dei richiami di fine fascicolo, alla reintroduzione della rigatura a secco, e cosi via ${ }^{32}$.

7. Per quanto concerne i testi degli autori classici è evidente che l'introduzione dell'antiqua, ha praticamente condotto alla scomparsa del modello del manoscritto cortese in gotica. Ma i manoscritti umanistici miniati della prima metà del secolo, e quelli fiorentini anche dopo, mostrano un fenomeno singolare: la persistenza nell'illustrazione dei testi classici dell'iconografica modemizzante tradizionale, per cui in essi, accanto alla scrittura rinnovata e imitata, compaiono gli dei, gli imperatori, i personaggi e i guerrieri vestiti salla moderna., o con strane fogge fantastiche, le città, i palazzi, le navi contemporanei.

Con ogni probabilità il fenomeno non è soltanto il frutto di una moda o della persistenza di una tradizione artigianale; ma del fatto che è l'immagine stessa del mondo classico che è rimasta legata a questi modelli gotici nella cultura e nella mentalità sia dei pittori che del pubblico: un pubblico che leggeva e preferiva la scrittura nuova; che gustava la nuova impaginazione e la rinnovata ornamentazione; ma che conservava ancora nella sua cultura iconografica le immagini proprie della stilizzazione gotica e dell'arte figurativa più tradizionali.

In particolare una serie di sondaggi forzatamente limitati mi hanno portato a formulare l'ipotesi che questa iconografia modernizzata o fantastica del mondo classico - tipica del gotico internazionale - si accompagnasse regolarmente all'uso delle maiuscole romaniche di stile toscano, e che ciò avvenisse naturalmente soprattutto nei codici di origine fiorentina, come, tanto per citare due Virgili, nel ms. Durazzo A VI 3 di Genova $^{33}$ e nel celebre Virgilio Riccardiano miniato da Apollonio di Giovanni e scritto da

"A. Derolez, Codicologie des manuscrits en écriture bumanistique sur parchemin, I, Texte. Tumhout 1984, pp. 164-166.

${ }^{33}$ Cf. D. Puncuh, I manoscritti della raccolta Durazzo, Genova 1979, pp. 134-136. 
Niccolò dei Ricci detto lo Spinoso ${ }^{34}$; ma ciò si verificava anche altrove, a Milano ${ }^{35}$, a Ferrara $^{36}$, nelle Marche ${ }^{37}$, a Roma ${ }^{38}$, e così via.

All'interno stesso della produzione manoscritta fiorentina di lusso del secolo XV si ha una sola eccezione a questa regola: eccezione costituita da un gruppo di codici contenenti testi classici eseguiti per il cardinale Giovanni d'Aragona dopo il 1480 e studiati assai recentemente da Albinia de la $\mathrm{Mare}^{39}$; in essi, mentre le maiuscole restavano di stile romanico-fiorentino, l'iconografia e la decorazione apparivano antichizzanti; in questo caso fu la preferenza personale del committente che provocò quella che la de la Mare ha definito la sunusual conjunction of script and decoration.40.

8. Ma nel Veneto già da quasi trent'anni lo stile delle maiuscole adoperate nell'epigrafia d'apparato, nei dipinti e infine anche nei codici si era modificato radicalmente secondo nuovi principi e nuovi (o meglio antichissimi) modelli. A Padova, dai primi anni Cinquanta del secolo, come ben si sa, Mantegna e Donatello avevano riprodotto la capitale delle iscrizioni romane e Felice Feliciano aveva ricostruito, per la prima volta, un Alpbabetum Romanum disegnato secondo l'susanza antichas ${ }^{11}$; in quell'am-

34 Facsimile integrale: Virgilius, Opera. Bucolica Georgica Aeneis. Manoscritto 492 della Biblioseca Riccardiana di Firenze, a cura di B. Maracchi Biagiarelli, Firenze 1969; ma cf. anche almeno P. e J. Courcelle, Lecteurs paĩens et lecteurs chrétiens de l'Enéide. 2. Les manuscrits illustrés de l'Enéide du $X^{e}$ au $X V^{e}$ sièche, Paris 1984, pp. 163-190 e figg. 342-353; e E. H. Gombrich, Apollomio di Giovanni. A Florentine cassone uorksbop seen through the eyes of a bumanist poet, in Id., Norm and Form, cit., pp. 11-28; per gli addobbi .fantastici. della maniera fiorentina, cf. A. Chastel, Les capitaines antiques affrontés dans l'art florentin du XVe siècke, in Id., Fables, Formes, Figures, I, Paris 1978, pp. 237-247; per il copista, cf. ora A. de la Mare, Research on Humanistic Scribes in Florence, in A. Garzelli, Miniatura fiorentina del Rinascimento. 1440-1525. Un primo censimento, 1, Firenze 1985 , n. 53, pp. 519-521.

3s Ove operavano il famoso maestro delle Vitae imperatorum, grande -modernizzatore, e to scriba Lorenzo Dolobella, per un cui Cesare, Vat. lat. 5824, cf. V. Brown, Portraits of Julius Caesar. in latin manuscripts of the Commentaries, in VIATOR, 12 (1981), pp. 340-341 e tav. 3, d.

${ }^{36}$ Un bell'Aulo Gellio modernizzato da Guglielmo Giraldi è nel noto ms. Ambrosiano SP Scotti, del 1448.

${ }^{37}$ A Fano sarebbe stato eseguito un Giustino del 1460, Ottob. lat. 1417: Degenhart - Schmitt, Corpus, cit., I, 1, Berlin 1968, n. 488 e tavv. 346-8.

${ }^{38} \mathrm{Cf}$. ad esempio il Cicero, Orationes, Vat. lat. 1742, scritto da Paolo di Middelburg fra il $1460 \mathrm{e}$ il 1470 , con Cicerone raffigurato come un professore universitario contemporaneo; e comunque cf. anche il materiale raccolto ed esposto da J. Ruysschaer, Miniaturistes sromainss sous Pie II, in Enea Silvio Piccolomini. Papa Pio II, Siena 1968, pp. 245-282.

${ }^{39}$ A. de la Mare, The Florentine Srribes of cardinal Giovanni of Aragon, in Il libro e il testo. Atti del Convegno internazionale, a cura di C. Questa - R. Raffaelli, Untino 1984, pp. 244-293 (con riproduzioni).

40 Ibid., p. 245.

"Cf. per questo, fra i tanti contributi, almeno quelli di M. Meiss, Toward a more comprebensive Renaissance Palaeography; in Art Bulletin, XLII (1960), pp. 97-112; E. Casamassima, Trattati di scrittura del Cinquecento italiano, Milano 1967, pp. 17-36; A. Petrucci, La scrittura. Ideologia $e$ rappresentazione, Torino 1986, pp. 21-28, con ampia bibliografia a PP. 170-171. 
biente $e$ in quella zona per la prima volta le capitali anticheggianti erano state introdotte direttamente nei manoscritti in funzione di scrittura d'apparato e di iniziali, probabilmente grazie ad una ardita trasposizione proposta da Bartolomeo Sanvito, il callig. rafo, o meglio il edesigner. più geniale del secolo ${ }^{42}$; iniziava così anche in campo grafico quel fenomeno che Carlo Dionisotti ha suggestivamente definito - un'evasione nel tempo, il pellegrinaggio umanistico ai santuari dell'antichità classica. ${ }^{43}$.

In realtà l'introduzione nel libro manoscritto di un alfabeto di tipo epigrafico e basato sull'imitazione diretta di modelli antichi rappresentava una vera e propria rivoluzione grafica, come ha sottolineato efficacemente Emanuele Casamassima: . Nel corso del secolo XV assistiamo per la prima volta nella storia della scrittura latina, con l'assunzione nel codice delle lettere lapidarie pure, alla contaminatio programmatica, più tardi all'accordo stilistico, cristallizzato nei tipi della stampa, di due forme distinte."4.

Il fenomeno conobbe nei manoscritti due differenti realizzazioni, rappresentate rispettivamente dalle lettere capitali dei titoli e dalle iniziali miniate, che venivano disegnate in modo tale da conferire alle singole lettere l'aspetto di caratteri incisi con sezione triangolare. I primi codici omati con tali lettere incise, possano essere considerati un Tolomeo eseguito a Monselice nel 1457 e donato dal govematore di Padova Giacomo Antonio Marcello a re Renato d'Angiò"s', e il famoso Strabone di Albi che Millard Meiss ha attribuito al Mantegna e che, più esattamente, J. Alexander propone invece oggi di assegnare a Bartolomeo Sanvito ${ }^{46}$. Nell'ambito di tale raffinato moto di renovatio antiquaria della scrittura capitale antica si colloca anche il fenomeno degli in. cipit, o dei veri e propri frontespizi, miniati in modo da raffigurare, o meglio da fingere, vere e proprie iscrizioni incise su lastre variamente inserite in una scena figurata, o sorrette da putti; un fenomeno tipico dei codici di lusso con testi classici e motivi ornamentali anticheggianti prodotti a Roma, nel Veneto, a Ferrara, ad Urbino, e cosi via.

In effetti il processo di renovatio grafica avviato dalla cultura antiquaria degli umanisti, dei pittori e dei calligrafi di Padova e del Veneto non si limitò ad introdurre nei libri manoscritti di lusso le lettere capitali epigrafiche e le iniziali incise. Tali lettere, infatti, erano veramente antiche, e rappresentavano una scrittura autenticamente -classicas; con esse un elemento vivo della cultura scritta del mondo romano entrava per la prima volta - nel libro del Rinascimento italiano. Assai naturalmente - e inevitabilmente, direi - un tale moto di vera e propria renovatio non poteva più sopportare,

12 Sul Sanvito, oltre la bibliografia cit. e il classico J. Wardrop. The Srript of Humanism. Some aspects of humanistic Script. 1460-1560, Oxford 1963, pp. 19-35, citerò solo due contributi recenti: Renaissance Painting in Manuscripts. Treasures from the British Library, a cura di T. Kren, New York 1983, pp. 103-106; M. L. Evans, Bartolommeo Sanvito and an antique motif, in The British Library Journal, XI (1985), pp. 123-130.

${ }^{4}$ C. Dionisotti, Geografia e storia della letteratura italiana, Torino 1967, p. 35.

4 Casamassima, Trattati, cit., p. 19.

4s Si tratta del Par. lat. 17542 segnalato in questo stesso volume da J. Alexander (cf. p. 148); sul ms. cf. anche Catalogue des manuscrits en écriture latine portant des indications de date, de lieu ou de copiste, III, Paris 1974, p. 583 e tav. CLXIV.

${ }^{16}$ M. Meiss, Andrea Mantegna as illuminator. An Episade in Renaissance Art, Humanism and Diplomacy, Hamburg 1957; e ora Alexander, in questo stesso volume, pp. 145-155. 
accanto ad una scrittura d'apparato autenticamente antica, la presenza di un'iconografia del mondo classico modernizzante, divenuta di colpo nel confronto insopportabilmente falsa ed anacronistica.

Si è già visto che nel codice umanistico di stile fiorentino si era instaurata una corrispondenza regolare fra l'uso delle capitali romaniche o toscane e l'iconografia modernizzante; con Mantegna, Sanvito e gli altri artisti e calligrafi della cultura antiquaria si scopre e si impone un altro e contrario rapporto: all'introduzione delle capitali epigrafiche romane nel libro manoscritto si fa corrispondere una radicale modificazione dell'immagine del mondo classico, dei suoi dei, dei suoi personaggi, dei suoi luoghi, dei suoi monumenti e costumi. L'instaurazione di tale rapporto non era stata ottenuta casualmente $o$ inconsapevolmente, ma costituiva il frutto di una precisa scelta culturale e stilistica; anche perché la diversità fra i differenti stili esecutivi, sia sul piano grafico, sia su quello iconografico, erano chiaramente individuati dai contemporanei, come dimostra una cedola della tesoreria aragonese che registra un pagamento effettuato il 16 febbraio del 1481 al miniatore Cristoforo Maiorana per aver eseguito in stile coerentemente antico. l'intera ornamentazione di un codice esopiano: sper lo principio ha facto con spiritello, animalii et altri lavuri antichi et in la lictera grande sta uno homo anticho che czappa uno jardino et altre lictere maiuscole ... E più per lo prezo de due lictere grande antiche. ${ }^{47}$; ove è evidente il rapporto coerente e cogente stabilito nella prassi artistica $e$ in quella amministrativa fra stile della scrittura d'apparato (elictere grande antiches), miniatura con iconografia autonoma (-homo antichos) e ornamentazione anticheggiante (slavuri antichis).

Secondo Panofsky e Saxl ait was the privilege of the Renaissance again to visualize classical subject matter under classical forms and so to reintegrate these two facts ${ }^{48}$.

9. Il confronto fra quanto è risultato dal presente studio e quanto si sa della storia del libro manoscritto italiano del Rinascimento fa ipotizzare che le modificazioni introdotte in Italia nel libro manoscritto di lusso verso la fine del Quattrocento con l'adozione delle capitali epigrafiche romane abbiano comportato una netta rottura con il passato e un globale rinnovamento del libro come prodotto, sia sotto l'aspetto propriamente grafico, sia sotto l'aspetto della struttura, del formato, dell'impaginazione, del rapporto fra testo e illustrazioni, della decorazione. In effetti, proprio durante questo processo nacque, grazie a Bartolomeo Sanvito, il libretto portatile (l'enchiridion) di Aldo Manuzio; e ancora, il carattere corsivo che chiamiamo italico passò dal manoscritto alla tipografia; inoltre, venne fissata la gerarchia delle scritture destinata a dominare la produzione del libro sino ad oggi: maiuscole epigrafiche di tipo classico, minuscole rotonde -romane, corsive italiche ${ }^{49}$; infine, proprio allora una interpretazione iconografica nuova, storica ed archeologica del mondo classico, s'affermò definitivamente.

${ }^{47}$ Cf. T. De Marinis, La biblioteca Napoletana dei re d'Aragona, II, Milano 1947, p. 273, doc. n. 592.

68 Panofsky - Saxl, Classical Mythology; cit., pp. 265-266.

49 Cf. per questo A. Petrucci, Alle origini del libro moderno: libri da banco, libri da bisaccia, li. bretti da mano, in Libri, scrittura e publico, cit., pp. 137-156. 
In queste pagine è stata troppo rapidamente riassunta una storia di più secoli, che ha vissuto almeno tre cambiamenti radicali dell'aspetto grafico, strutturale ed iconografico dei manoscritti di lusso di autori classici. Ciascuna volta è possibile individuare una precisa e diretta corrispondenza fra questi cambiamenti e alcune profonde modificazioni della cultura contemporanea, $e$ in particolare delle pratiche di studiare, di leggere, di produrre libri. Infine il processo si è concluso, fra XV e XVI secolo, con la fissazione definitiva del modello grafico, strutturale ed iconografico del libro moderno.

lo ho l'impressione che Guglielmo Cavallo avesse dunque ragione di affermare che .il percorso che va da un campo di tensioni letterario a un campo di tensioni grafico [e, si può aggiungere, iconografico] fa dell'età umanistica ... un punto nodale nella storia della tradizione manoscritta; ed un punto nodale di cui, più di altri, viviamo tuttora, in positivo o in negativo, le conseguenze. ${ }^{\text {so }}$.

${ }^{\text {so }} \mathrm{G}$. Cavallo, Frammenti di un discorso grafico-textuale, in $1 /$ libro e il testo, cit., pp. 415-429; la citazione da p. 429. 